\title{
Design of Finite-Time Synchronization Controller and Its Application to Security Communication System
}

\author{
Tao Ren*, Zhi-liang Zhu* and Hai Yu \\ Software College, Northeastern University, Shenyang Liaoning 110004, P R China
}

Received: 7 Jul. 2013, Revised: 17 Nov. 2013, Accepted: 20 Nov. 2013

Published online: 1 Jan. 2014

\begin{abstract}
In this paper, an finite-time synchronization controller is proposed for the general chaos model. Compared to other results, the controller designed is simpler and independent on the nonlinear function of chaos system. So the finite-time control scheme can be used in a wide range of chaos system. The simulation results are given to illustrate the effectiveness of the finite-time controller and the applicability to security communication system of this synchronization scheme.
\end{abstract}

Keywords: Chaos system, synchronization, finite-time control, security communication.

\section{Introduction}

Chaos synchronization has been a focused research topic during the last decade due to its theoretical and practical applications especially in security communication. Many schemes and techniques for controlling chaos synchronization such as adaptive feedback control [1-4], state feedback control [5, 6] and observer-based approach [7] etc have been developed. Furthermore, some attractive results of synchronization and applications in secure communication for a class of chaotic systems have been reported [1-16].

Based on the above results, a large number of chaos synchronization schemes are aimed at asymptotical stability of error synchronization dynamics. From the practical point of view, however, it is more valuable that the synchronization objective is realized in a finite time. Especially in secure communication systems for instance, the range of time during which chaotic systems are out of synchrony is equivalent to the range of time in which the encoded message/data cannot be recovered or sent. Therefore, the synchronization time optimization is essential for satisfying the requirement of communication and this could be done by means of finite-time control-a very promising technique by which we can decide the settling time flexibly. Some authors have investigated chaos synchronization based on finite-time recently [8-16]. However, most of the reported works are more specific to some systems, such as Lorenz system and Chua system. There are some limitations for these contributions to be applied to the other systems except the one studied. The other problem for some works is the complex controller design. Some controllers proposed contain all or parts of the nonlinear function, and then the closed-loop error system can be deduced to linear system. This method will reduce the difficulties of mathematical proof, meanwhile make the controller too complex compared to systems being controlled. These approaches are usually difficult to implement in experiments when possible.

Motivated by the above discussion, in this paper, a simple synchronization controller is proposed for realizing synchronization in finite time based on the general chaos system. The structure of the controller is independent on the chaos system, so it can be implemented in many chaos systems. The numerical simulations are given to illustrate the effectiveness.

The rest of the paper is organized as follows. In Section 2 , the finite-time controller is designed to guarantee the stability of the error system. In Section 3, the simulation example is given to illustrate the applicability for security communication of the proposed approach. A conclusion is drawn in the last section.

Notations: $I$ denotes the identity matrix of appropriate dimensions. $\|x(t)\|$ means the Euclidean vector norm at time $t$, while $\|x(t)\|_{2}:=\sqrt{\int_{0}^{\infty}\|x(t)\|^{2} \mathrm{~d} t} \cdot \operatorname{sgn}(\cdot)$ is denoted

\footnotetext{
*Corresponding author e-mail: chinarentao@163.com, zzl@ mail.neu.edu.cn
} 
as sign function. $\operatorname{diag}(\cdots)$ represents a block-diagonal matrix.

\section{The Main Results}

Consider the following master-slave synchronization system

$$
\begin{aligned}
& \mathrm{M}: \dot{x}(t)=A x(t)+f(x(t)) \\
& \mathrm{S}: \dot{y}(t)=A y(t)+f(y(t))+u(t)
\end{aligned}
$$

where $\mathrm{M}$ and $\mathrm{S}$ denote the master system and slave system with the state vectors $x(t) \in R^{n}$ and $y(t) \in R^{n}$ respectively. $A$ is the constant matrix with appropriate dimensions. $u(t)=\left(u_{1}(t), \cdots, u_{n}(t)\right)$ is the finite-time controller to be designed.

Defining $e(t)=y(t)-x(t)$, then we have the error dynamic system as follows

$$
\dot{e}(t)=A e(t)+f(y(t))-f(x(t))+u(t)
$$

The controller $u(t)$ is proposed as:

$$
u_{i}(t)=-k\left|e_{i}(t)\right|^{\alpha} \operatorname{sgn}\left(e_{i}(t)\right)
$$

where constant $k$ is the controller gain and the constant $\alpha \in(0,1)$.

Fact 1 According to the features of chaos system, each state of chaos system $\dot{x}(t)=f(x(t))$ is bounded, furthermore the error of any two states is bounded too.

From Fact 1 we can get the conclusion that there exists a constant $M \geq 0$, such that the state of error system (2) satisfies $\|e\|^{2} \leq M$.

Assumption 1 The nonlinear function $f: R^{n} \rightarrow R^{n}$ in (1) satisfies the following Lipschitz condition:

$$
\left\|f\left(x_{1}\right)-f\left(x_{2}\right)\right\| \leq \gamma\left\|x_{1}-x_{2}\right\|
$$

where $\gamma$ is a positive scalar.

Lemma 1 Suppose $a_{1}, a_{2}, \cdots, a_{n}$ and $0<q<2$ are all real numbers, then the following inequality holds:

$$
\left|a_{1}\right|^{q}+\left|a_{2}\right|^{q}+\cdots+\left|a_{n}\right|^{q} \geq\left(a_{1}^{2}+a_{2}^{2}+\cdots+a_{n}^{2}\right)^{q / 2} .
$$

Lemma $2^{[15]}$ Consider the system

$$
\dot{x}(t)=f(x(t)), \quad f(0)=0, x \in R^{n}
$$

where $f: D \rightarrow R^{n}$ is continuous on an open neighborhood $D \subset R^{n}$. Suppose there exists a continuous differential positive-definite function $V(x(t)): D \rightarrow R$, real numbers $p>0,0<\eta<1$, such that

$$
\dot{V}(x(t))+p V^{\eta}(x(t)) \leq 0, \forall x(t) \subset D
$$

Then, the origin of system (5) is a locally finite-time stable equilibrium, and the settling time, depending on the initial state $x(0)=x_{0}$, satisfies

$$
T_{1} \leq \frac{V^{1-\eta}\left(x_{0}\right)}{p(1-\eta)}
$$

In addition, if $D=R^{n}$ and $V(x(t))$ is also radially unbounded (i.e. $V(x(t)) \rightarrow \infty$ as $x(t) \rightarrow \infty$ ), then the origin is a globally finite-time stable equilibrium of system (5).

Theorem 1. The error system (2) can achieve stability (i.e. the synchronization of the master and slave system (1) is achieved) in finite time $T_{1}$ by the controller (3), if there exists constant $k \geq\left(d+\lambda M^{\frac{1-\alpha}{2}}\right) / 2>0$, the settling time $T_{1}$ is determined by

$$
T_{1} \leq \frac{2\left(V\left(e_{0}\right)\right)^{\frac{1-\alpha}{2}}}{d(1-\alpha)} .
$$

where $\lambda=\max \left\{0, \lambda_{\max }\right\}$, in which $\lambda_{\max }$ is the maximum eigenvalue of $2(A+\gamma I)$ and $\gamma$ is Lipschitz constant of $f(\cdot)$, constant $d>0, \alpha \in(0,1)$ and $M \geq 0$ such that $\|e\|^{2} \leq M$.

Proof: Construct a Lyapunov function candidate as

$$
V(e)=\left(e_{1}^{2}+e_{2}^{2}+\ldots+e_{n}^{2}\right)
$$

Taking the time derivative of $V(t)$ and substituting (2) and (3) into $\dot{V}(t)$ yield

$$
\begin{aligned}
\dot{V}(e)= & 2\left(\dot{e}_{1} e_{1}+\dot{e}_{2} e_{2}+\ldots \dot{e}_{n} e_{n}\right) \\
& =2 e^{\mathrm{T}} A e+2 \sum_{i=1}^{n}\left(f_{i}(y)-f_{i}(x)+u_{i}\right) \cdot e_{i} \\
& \left.\leq 2 e^{\mathrm{T}} A e+2 \sum_{i=1}^{n}\left\|f_{i}(y)-f_{i}(x)\right\| \cdot\left\|e_{i}\right\|\right) \\
& -2 k \sum_{i=1}^{n}\left|e_{i}\right|^{\alpha+1}
\end{aligned}
$$

by virtue of Assumption 1, we have

$$
\begin{aligned}
\dot{V}(e) & \leq 2 e^{\mathrm{T}} A e+2 e^{\mathrm{T}} \gamma e-2 k \sum_{i=1}^{n}\left|e_{i}\right|^{\alpha+1} \\
& =2 e^{\mathrm{T}} A+\gamma I e-2 k \sum_{i=1}^{n}\left(\left|e_{i}\right|^{2}\right)^{\frac{\alpha+1}{2}} \\
& \leq \lambda_{\max } e^{\mathrm{T}} e-2 k \sum_{i=1}^{n}\left(\left|e_{i}\right|^{2}\right)^{\frac{\alpha+1}{2}}
\end{aligned}
$$

Due to the fact $0.5<\frac{\alpha+1}{2}<1$ and Lemma 1 , we get

$$
\begin{aligned}
\dot{V}(e) \leq & \lambda_{\max } e^{\mathrm{T}} e-2 k(V(e))^{\frac{\alpha+1}{2}} \\
& =-\left(2 k-\lambda_{\max }(V(e))^{\frac{1-\alpha}{2}}\right)(V(e))^{\frac{\alpha+1}{2}}
\end{aligned}
$$

By Theorem 1 , we can get $k \geq\left(d+\lambda M^{\frac{1-\alpha}{2}}\right) / 2>0$, then

$$
\dot{V}(e) \leq-\left(d+\lambda M^{\frac{1-\alpha}{2}}-\lambda_{\max }(V(e))^{\frac{1-\alpha}{2}}\right)(V(e))^{\frac{\alpha+1}{2}}
$$

Next, we will discuss the value of $\dot{V}(e)$ according to $\lambda_{\text {max }}$. There are two possibilities for $\lambda_{\text {max }}$ : 
(1) When $\lambda_{\max } \leq 0$, then $\lambda=0$ due to the fact that $\lambda=\max \left\{0, \lambda_{\max }\right\}$, it yeilds

$$
\begin{gathered}
\dot{V}(e) \leq- \\
\left(d-\lambda_{\max }(V(e))^{\frac{1-\alpha}{2}}\right)(V(e))^{\frac{\alpha+1}{2}} \\
\leq-d(V(e))^{\frac{\alpha+1}{2}}
\end{gathered}
$$

(2) When $\lambda_{\max }>0$, then $\lambda=\lambda_{\max }$, one can get

$$
\dot{V}(e) \leq-\left(d+\lambda_{\max }\left(M^{\frac{1-\alpha}{2}}-(V(e))^{\frac{1-\alpha}{2}}\right)\right)(V(e))^{\frac{\alpha+1}{2}}
$$

According to Fact 1, we have the following inequality $V(e)=\sum_{i=1}^{n} e_{i}^{2}=\|e\|^{2} \leq M$, it yields

$$
\dot{V}(e) \leq-d(V(e))^{\frac{\alpha+1}{2}}
$$

Therefore, no matter what the value of $\lambda_{\max }$ is, we can conclude that $\dot{V}(e) \leq-d(V(e))^{\frac{\alpha+1}{2}}$, in which $d>0, \alpha \in$ $(0,1)$. According to Lemma 2, we can get the conclusion that the error dynamic can achieve stability in finite time and the settling time $T_{1} \leq 2\left(V\left(e_{0}\right)\right)^{\frac{1-\alpha}{2}} / d(1-\alpha)$. Hence the chaos system (2) can synchronize before time $T_{1}$. This completes the proof.

\section{Numerical Simulation}

In this section, the following Lorenz systems are considered as master and slave system for the security communication scheme based on chaos masking scheme.

$$
\begin{gathered}
\text { Master system: }\left\{\begin{array}{l}
\dot{x}_{1}=a\left(x_{2}-x_{1}\right) \\
\dot{x}_{2}=c x_{1}-x_{2}-x_{1} x_{3} \\
\dot{x}_{3}=x_{1} x_{2}-b x_{3}
\end{array}\right. \\
\text { Slave system: }\left\{\begin{array}{l}
\dot{y}_{1}=a\left(y_{2}-y_{1}\right)+u_{1} \\
\dot{y}_{2}=c y_{1}-y_{2}-y_{1} y_{3}+u_{2} \\
\dot{y}_{3}=y_{1} y_{2}-b y_{3}+u_{3}
\end{array}\right.
\end{gathered}
$$

where $a=10, b=8 / 3, c=28$. We set the initial value for both chaos systems as $\left(x_{1}(0), x_{2}(0), x_{3}(0)\right)=(1,2,0)$ and $\left(y_{1}(0), y_{2}(0), y_{3}(0)\right)=(0,1,2)$, then the initial value of error system is $\left(e_{1}(0), e_{2}(0), e_{3}(0)\right)=(-1,-1,2)$.

As is shown in Figure 1 and 2, the three states of Lorenz system are bounded independent on the initial value, i.e. $x(t) \in(-20,20), \quad y(t) \in(-30,30)$, $z(t) \in(0,50)$, so are the error state values. Due to Fact 1 $\|e\|^{2} \leq M$, we chose $M=7700$ for Lorenz system. The Lipschitz constant is calculated as $\gamma=65$.

Then we can get $\lambda_{\max }=147$ by calculating the maximum eigenvalue of $2(A+\gamma I)$. Hence, the range of the controller gain is $k \geq 182$, we chose $k=185$ for simulation. Meanwhile, we set the parameters $d=4$ and $\alpha=0.8$, by Theorem 1 the settling time is calculated as $T_{1} \leq 2.99 \mathrm{~s}$. Figure 3 shows that the states of error dynamic can regulate to zero in short time, less than the settling time $T_{1}$. This implies that the master and slave
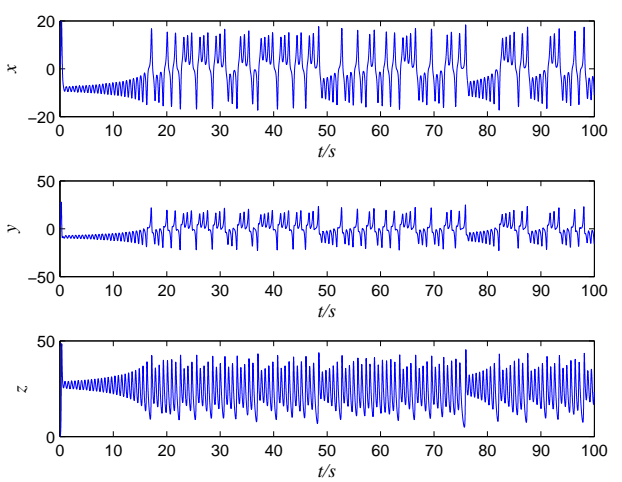

Fig. 1: The three state trajectories of Lorenz system

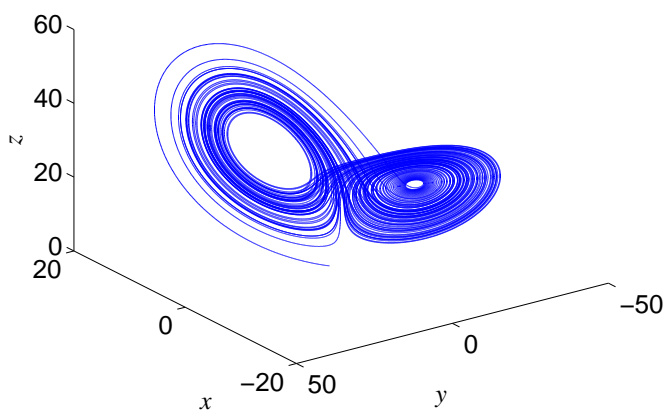

Fig. 2: The chaotic attractors of Lorenz system
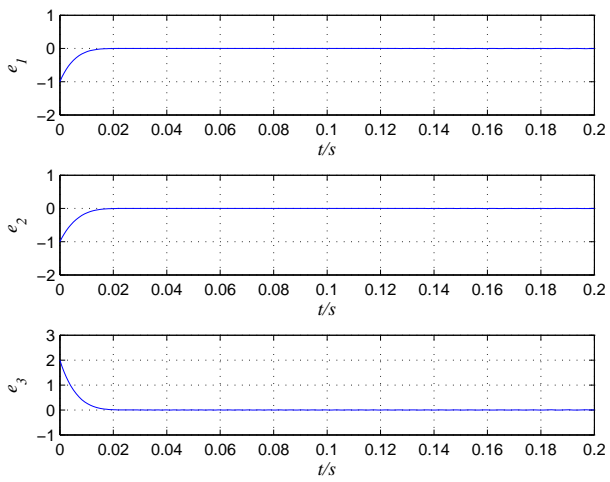

Fig. 3: The state trajectories of error system 
chaos systems can synchronize by the finite-time control scheme proposed very quickly.

The initial Sine wave signal can be masked well by the master system shown in Figure 4, the transmitted signal looks like noise signal and impossibly attracts attention of intruder. The recovered signal is shown in the bottom half of Figure 5, which is almost the same as the initial Sine wave signal shown in the top half of the same Figure.

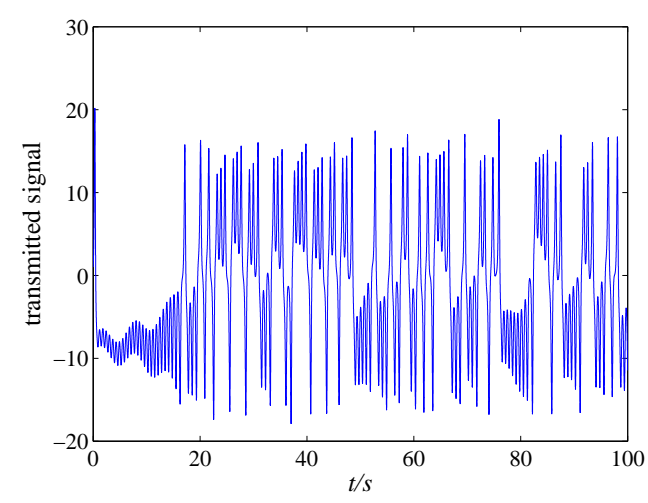

Fig. 4: The transmitted/masked signal
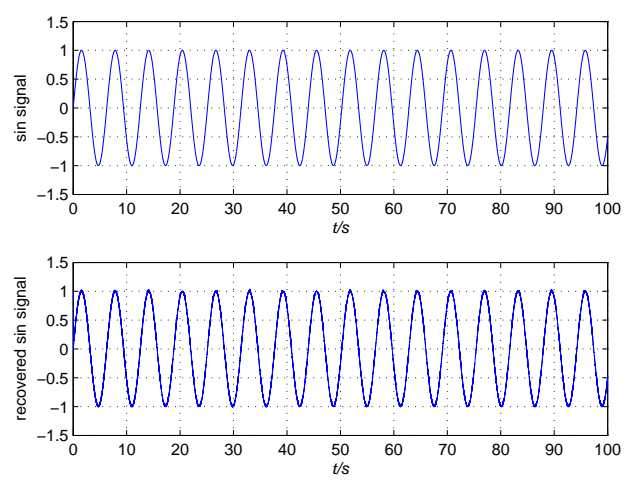

Fig. 5: The Sine signal (initial signal is in the top half; recovered signal is in the bottom half)

So the security communication scheme based on chaos synchronization can not only protect the message from attacking by the intruder but provide a good way to recover the useful signal by the receiver.

\section{Conclusion}

In this paper, control theory is used to formalize finite-time chaos synchronization controller. The stability criteria is presented for error system based on Lyapunov method. Compared to other results, the proposed controller is simpler. The numerical simulation results for Lorenz system show the effectiveness of our finite-time control scheme and its applicability to security communication system. The scheme provides a safe way to mask useful information carrying signal.

\section{Acknowledgement}

The authors acknowledge the financial support by the National Natural Science Foundation of China (Grant No. 61104074), the China Postdoctoral Science Foundation (Grant No. 2013T60294, 20100471462), the Fundamental Research Funds for the Central Universities of Ministry of Education of China (Grant No. N100317002, N100604007, N110417004, N110417005, N110617001) and the Scientific Research Foundation for Doctor of Liaoning Province, China (Grant No. 20101032). The author is grateful to the anonymous referee for a careful checking of the details and for helpful comments that improved this paper.

\section{References}

[1] J. H. Park, S. M. Lee, O. M. Kwon, Phys. Lett., A371, 263 (2007).

[2] M. F. Hu and Z. Y. Xu, Chinese Physics, 16, 3231 (2007).

[3] J. H. Park, Int. J. Nonlinear Sci. Numer. Simul., 6, 201 (2005).

[4] H. Salarieh and A. Alasty, Commun Nonlinear Sci Numer Simul., 14, 508 (2009).

[5] H. H. Chen, G. J. Sheu, Y. L. Lin and C. S. Chen, Nonlinear Analysis: Theory, Methods and Applications, 70, 4393 (2009).

[6] H. H. Chen, Physics Letters, A372, 1841 (2008).

[7] J. Meng and X. Y. Wang, Physics Letters, A369, 294 (2007).

[8] S. H. Li and Y. P. Tian, Chaos, Solitons and Fractals, 15, 303 (2003).

[9] N. Cai, Y. W. Jing and S. Y. Zhang, Commun Nonlinear Sci Numer Simulat, 15, 1613 (2010).

[10] P. Wilfrid, F. Thierry and M. Emmanuel, IEEE Transactions on Automatic Control, 53, 356 (2008).

[11] H. Wang, Z. Z. Han, Q. Y. Xie and W. Zhang, Commun Nonlinear Sci Numer Simulat, 14, 2239 (2009).

[12] H. Wang, Z. Z. Han, Q. Y. Xie and W. Zhang, Commun Nonlinear Sic Numei Simulat, 14, 2728 (2009).

[13] H. Wang, Z. Z. Han, Q. Y. Xie and W. Zhang, Nonlinear Analysis: Real World Applications, 10, 2842 (2009).

[14] U. E. Vincent and R. Guo, Physics Letters, A375, 2322 (2011).

[15] M. P. Aghababa, S. Khanmohammadi and G. Alizadeh, Applied Mathematical Modelling, 35, 3080 (2011).

[16] W. G. Yu, Physics Letters, A374, 3021 (2010). 


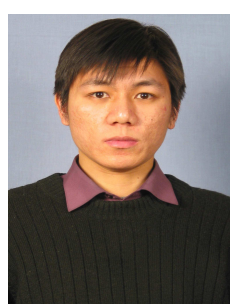

Tao Ren was born in 1980 in China and received the $\mathrm{PhD}$ degree from Northeastern University in control theory and control engineering in 2007. $\mathrm{He}$ is currently an associate professor in software college, Northeastern University. His main research interests include chaos synchronization control, chaos security communication, congestion control of high speed computer networks. He has published more than 30 papers and one book. He is now responsible for 7 academic research projects in China.

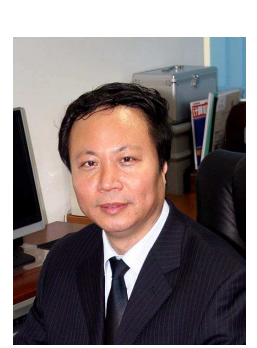

\section{Zhi-liang}

Zhu received the $\mathrm{PhD}$ degree in computer science from Northeastern University in 2002. His main research interests include information integrate, complexity software system, network coding and communication security, chaos-based digital communications, applications of complex-network theories, and cryptography. By far, he has authored and co-authored over 130 international journal papers and 100 conference papers. Additionally, he published 5 books, including Introduction to Communication and Program Designing of Visual Basic .NET. He is also the recipient of 9 academic awards at the national, ministerial and provincial level.

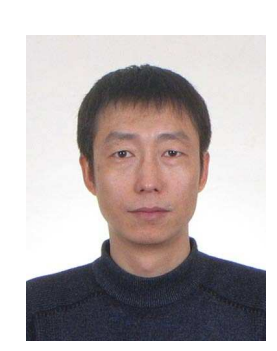

Hai Yu received the $\mathrm{PhD}$ degree in Computer Software and Theory in 2006 at the Northeastern University. From May 2009 to August 2009, he worked shortly as a Senior Research Assistant to the Centre for Chaos and Complex Networks, Department of Electronic Engineering, City University of Hong Kong, under the supervision of Prof. Guanrong Chen. His research interests have focused mainly on multimedia security and secure chaos-based communications, encompassing Digital Watermarking, Information Hiding, Video Coding, Digital Chaotic Cipher, Network Coding, Channel Coding and applications of complex-network theories to communications. 